\title{
A noncommutative symmetric system over the Grossman-Larson Hopf algebra of labeled rooted trees
}

\author{
Wenhua Zhao
}

Received: 23 April 2007 / Accepted: 30 August 2007 / Published online: 25 September 2007

(C) Springer Science+Business Media, LLC 2007

\begin{abstract}
In this paper, we construct explicitly a noncommutative symmetric $(\mathcal{N C S})$ system over the Grossman-Larson Hopf algebra of labeled rooted trees. By the universal property of the $\mathcal{N}$ CS system formed by the generating functions of certain noncommutative symmetric functions, we obtain a specialization of noncommutative symmetric functions by labeled rooted trees. Taking the graded duals, we also get a graded Hopf algebra homomorphism from the Connes-Kreimer Hopf algebra of labeled rooted forests to the Hopf algebra of quasi-symmetric functions. A connection of the coefficients of the third generating function of the constructed $\mathcal{N C S}$ system with the order polynomials of rooted trees is also given and proved.
\end{abstract}

Keywords Noncommutative symmetric functions · Grossman-Larson Hopf algebra · Connes-Kreimer Hopf algebras · Labeled rooted trees

\section{Introduction}

Let $K$ be any unital commutative $\mathbb{Q}$-algebra and $A$ a unital associative but not necessarily commutative $K$-algebra. Let $t$ be a formal central parameter, i.e. it commutes with all elements of $A$, and $A[[t]]$ the $K$-algebra of formal power series in $t$ with coefficients in $A$. A $\mathcal{N} C S$ (noncommutative symmetric) system over $A$ (see Definition 2.1) by definition is a 5-tuple $\Omega \in A[[t]]^{\times 5}$ which satisfies the defining equations (see Eqs. (2.1)-(2.5)) of the NCSFs (noncommutative symmetric functions) first introduced and studied in the seminal paper [10]. When the base algebra $K$ is clear in the context, the ordered pair $(A, \Omega)$ is also called a $\mathcal{N} C S$ system. In some sense,

W. Zhao $(\bowtie)$

Department of Mathematics, Illinois State University, Normal, IL 61790-4520, USA

e-mail:wzhao@ilstu.edu 
a $\mathcal{N C S}$ system over an associative $K$-algebra can be viewed as a system of analogs in $A$ of the NCSFs defined by Eqs. (2.1)-(2.5). For some general discussions on the $\mathcal{N C S}$ systems, see [28]. For a family of $\mathcal{N C S}$ systems over differential operator algebras and their applications to the inversion problem, see [29] and [30]. For more studies on NCSFs, see [6, 16-18, 25] and [5].

One immediate but probably the most important example of the $\mathcal{N}$ CS systems is $(\mathcal{N} S y m, \Pi)$ formed by the generating functions of the NCSFs defined in [10] by Eqs. (2.1)-(2.5) over the free $K$-algebra $\mathcal{N} S y m$ of NCSFs (see Sect. 2). It serves as the universal $\mathcal{N C S}$ system over all associative $K$-algebra (see Theorem 2.6). More precisely, for any $\mathcal{N}$ CS system $(A, \Omega)$, there exists a unique $K$-algebra homomorphism $\mathcal{S}: \mathcal{N} S y m \rightarrow A$ such that $\mathcal{S}^{\times 5}(\Pi)=\Omega$ (here we have extended the homomorphism $\mathcal{S}$ to $\mathcal{S}: \mathcal{N} \operatorname{Sym}[[t]] \rightarrow A[[t]]$ by the base extension).

The universal property of the $\mathcal{N}$ CS system $(\mathcal{N} S y m, \Pi)$ can be applied as follows when a $\mathcal{N} C S$ system $(A, \Omega)$ is given. Note that, as an important topic in the symmetric function theory, the relations or polynomial identities among various NCSFs have been worked out explicitly (see [10]). When we apply the $K$-algebra homomorphism $\mathcal{S}: \mathcal{N} S y m \rightarrow A$ guaranteed by the universal property of the system $(\mathcal{N} S y m, \Pi)$ to these identities, they are transformed into identities among the corresponding elements of $A$ in the system $\Omega$. This will be a very effective way to obtain identities for certain elements of $A$ if we can show they are involved in a $\mathcal{N} C S$ system over $A$. On the other hand, if a $\mathcal{N} C S$ system $(A, \Omega)$ has already been well-understood, the $K$-algebra homomorphism $\mathcal{S}: \mathcal{N} S y m \rightarrow A$ in return provides a specialization or realization $[10,24]$ of NCSFs, which may provide some new understandings on NCSFs. For more studies on the specializations of NCSFs, see the references quoted above for NCSFs.

In this paper, we apply the gadget above to the Grossman-Larson Hopf algebra of labeled rooted trees. To be more precise, for any non-empty $W \subseteq \mathbb{N}^{+},{ }_{1}^{1}$ let $\mathcal{H}_{G L}^{W}$ be the Grossman-Larson Hopf algebra $\mathcal{H}_{G L}^{W}[4,8,9,12]$ of rooted trees labeled by positive integers of $W$. We first introduce five generating functions (see Eqs. (4.1)(4.4) and Eq. (4.15)) of certain elements of $\mathcal{H}_{G L}^{W}$ and show that they form a $\mathcal{N} C S$ system $\Omega_{\mathbb{T}}^{W}$ over $\mathcal{H}_{G L}^{W}$ (see Theorem 4.5). Then, by the universal property of the $\mathcal{N} C S$ system $(\mathcal{N} S y m, \Pi)$ from NCSFs, we obtain a graded Hopf algebra homomorphism $\mathcal{T}_{W}: \mathcal{N} S y m \rightarrow \mathcal{H}_{G L}^{W}$, which gives a specialization of NCSFs by $W$-labeled rooted trees (see Theorem 4.6). By taking the graded duals, we get a graded Hopf algebra homomorphism $\mathcal{T}_{W}^{*}: \mathcal{H}_{C K}^{W} \rightarrow \mathcal{Q S y m}$ from the Connes-Kreimer Hopf algebra $\mathcal{H}_{C K}^{W}$ $[4,8,9,15]$ to the Hopf algebra $\mathcal{Q} S y m$ of quasi-symmetric functions [11, 20, 24]. Later in [31], it will be shown that, when $W=\mathbb{N}^{+}$, the specialization $\mathcal{T}_{W}$ above is actually an embedding and hence the Hopf algebra homomorphism $\mathcal{T}_{W}^{*}$ is onto. Finally, we give a combinatorial interpretation of the constants $\theta_{T}$ (see Definition 4.2) of rooted trees $T$ that appeared in the third component $\tilde{d}(t)$ (see Eq. (4.15)) of the $\mathcal{N C S}$ system $\Omega_{\mathbb{T}}^{W}$ above. We show that, for each rooted tree $T$, the constant $\theta_{T}$

\footnotetext{
${ }^{1}$ All constructions and results of this paper work equally well for any non-empty weighted set $W$, i.e. any non-empty set $W$ with a fixed weight function $w t: W \rightarrow \mathbb{N}^{+}$such that, for any $k \in \mathbb{N}^{+}, w t^{-1}(k)$ is a finite subset of $W$. But, for simplicity and convenience, we will always assume that $W$ is a non-empty subset of $\mathbb{N}^{+}$.
} 
coincides with the coefficient of $s$ in the order polynomial $\Omega\left(B_{-}(T), s\right)$ (see [24]), where $B_{-}(T)$ is the rooted forest obtained by cutting off the root of $T$.

The arrangement of the paper is as follows. In Sect. 2, we first recall the definition of the $\mathcal{N}$ CS systems [28] $\Omega$ over the $K$-algebras $A$ and a result (see Proposition 2.2) on the $\mathcal{N}$ CS systems when $A$ is further a bialgebra or Hopf algebra. We then recall the universal $\mathcal{N} C S$ system $(\mathcal{N} S y m, \Pi)$ formed by generating functions of certain NCSFs in [10]. The Hopf algebra structure of $\mathcal{N} S y m$ and the universal property of the $\mathcal{N}$ CS system $(\mathcal{N} S y m, \Pi)$ (see Theorem 2.6) will also be reviewed.

In Sect. 3, we first fix certain notation on rooted trees and recall the ConnesKreimer Hopf algebra $\mathcal{H}_{C K}^{W}$ and the Grossman-Larson Hopf algebra $\mathcal{H}_{G L}^{W}$ of $W$ labeled rooted forests and rooted trees, respectively. Then, by using the duality between the Grossman-Larson Hopf algebra and the Connes-Kreimer Hopf algebra (see Theorem 3.2), we prove a technic lemma, Lemma 3.4, that will be crucial for our later arguments.

In Sect. 4, we introduce five generating functions of $W$-labeled rooted trees and show in Theorem 4.5 that they form a $\mathcal{N C S}$ system $\Omega_{\mathbb{T}}^{W}$ over the Grossman-Larson Hopf algebra $\mathcal{H}_{G L}^{W}$. By the universal property of the system $(\mathcal{N} S y m, \Pi)$, we get a graded $K$-Hopf algebra homomorphism $\mathcal{T}_{W}: \mathcal{N} S y m \rightarrow \mathcal{H}_{G L}^{W}$ (see Theorem 4.6). By taking the graded duals, we get a graded Hopf algebra homomorphism $\mathcal{T}_{W}^{*}: \mathcal{H}_{C K}^{W} \rightarrow$ $\mathcal{Q} S y m$ from the Connes-Kreimer Hopf algebra $\mathcal{H}_{C K}^{W}$ to the Hopf algebra $\mathcal{Q} S y m$ of quasi-symmetric functions (see Corollary 4.7).

In Sect. 5, we first recall the strict order polynomials and the order polynomials of finite posets (partially ordered sets). Then, by applying some of results proved in [26] for the strict order polynomials of rooted forests and the well-known Reciprocity Relation (see Proposition 5.1) between the strict order polynomials and the order polynomials of finite posets, we show in Proposition 5.8 that, for any $T \in \overline{\mathbb{T}}$, the constant $\theta_{T}$ involved in the third component of the $\mathcal{N C S}$ system $\Omega_{\mathbb{T}}^{W}$ is same as the coefficient of $s$ of the order polynomial $\Omega_{(}\left(B_{-}(T), s\right)$ of the rooted forest $B_{-}(T)$.

Finally, two remarks are as follows. First, as we pointed out early, by applying the specialization $\mathcal{T}_{W}: \mathcal{N} S y m \rightarrow \mathcal{H}_{G L}^{W}$, we will get a host of identities for the rooted trees involved in the $\mathcal{N C S}$ system $\Omega_{\mathbb{T}}^{W}$ from the identities of the NCSFs in $\Pi$. We believe some of these identities are interesting, at least from a combinatorial point view. But, to keep this paper in a certain size, we have to ask the reader who is interested to do the translations via the Hopf algebra homomorphism $\mathcal{T}_{W}: \mathcal{N} S y m \rightarrow$ $\mathcal{H}_{G L}^{W}$. Secondly, some relations between the $\mathcal{N}$ CS system $\left(\mathcal{H}_{G L}^{W}, \Omega_{\mathbb{T}}^{W}\right)$ constructed in this paper and the $\mathcal{N C S}$ systems constructed in [29] over differential operator algebras will be further studied in the followed paper [31]. Some consequences of those relations to the inversion problem ([3] and [7]) and specializations of NCSFs will also be derived there. In particular, it will be shown that, with the label set $W=$ $\mathbb{N}^{+}$, the $K$-Hopf algebra homomorphism $\mathcal{T}_{W}: \mathcal{N} S y m \rightarrow \mathcal{H}_{G L}^{W}$ in Theorem 4.6 is actually an embedding.

\section{The universal $\mathcal{N}$ CS system from noncommutative symmetric functions}

In this section, we first recall the definition of the $\mathcal{N C S}$ systems [28] over associative algebras and some of the NCSFs (noncommutative symmetric functions) first intro- 
duced and studied in the seminal paper [10]. We then discuss the universal property of the $\mathcal{N}$ CS system formed by the generating functions of these NCSFs. The main result that we will need later is Theorem 2.6 which was proved in [28]. For some general discussions on the $\mathcal{N}$ CS systems, see [28]. For more studies on NCSFs, see $[6,16-18,25]$ and [5].

Let $K$ be any unital commutative $\mathbb{Q}$-algebra ${ }^{2}$ and $A$ any unital associative but not necessarily commutative $K$-algebra. Let $t$ be a formal central parameter, i.e. it commutes with all elements of $A$, and $A[[t]]$ the $K$-algebra of formal power series in $t$ with coefficients in $A$. First let us recall the following main notion of this paper.

Definition 2.1 ([28]) For any unital associative $K$-algebra $A$, a 5-tuple $\Omega=(f(t)$, $g(t), d(t), h(t), m(t)) \in A[[t]]^{\times 5}$ is said to be a $\mathcal{N} C S$ (noncommutative symmetric) system over $A$ if the following equations are satisfied.

$$
\begin{aligned}
& f(0)=1, \\
& f(-t) g(t)=g(t) f(-t)=1, \\
& e^{d(t)}=g(t), \\
& \frac{d g(t)}{d t}=g(t) h(t), \\
& \frac{d g(t)}{d t}=m(t) g(t) .
\end{aligned}
$$

When the base algebra $K$ is clear in the context, we also call the ordered pair $(A, \Omega)$ a $\mathcal{N} C S$ system. Since $\mathcal{N} C S$ systems often come from generating functions of certain elements of $A$ that are under the consideration, the components of $\Omega$ will also be refereed as the generating functions of their coefficients.

All $K$-algebras $A$ that we are going to work on in this paper are $K$-Hopf algebras. We will freely use some standard notions and results from the theory of bialgebras and Hopf algebras, which can be found in the standard text books [1, 14] and [21]. For example, by a sequence of divided powers of a bialgebra or Hopf algebra $A$ we mean a sequence $\left\{c_{n} \mid n \geq 0\right\}$ of elements of $A$ such that, for any $n \geq 0$, we have

$$
\Delta c_{n}=\sum_{k \geq 0} c_{k} \otimes c_{n-k} .
$$

The following result proved in [28] later will be useful to us.

Proposition 2.2 Let $(A, \Omega)$ be a $\mathcal{N} C S$ system as above. Suppose $A$ is further a $K$-bialgebra. Then the following statements are equivalent.

(a) The coefficients of $f(t)$ form a sequence of divided powers of $A$.

(b) The coefficients of $g(t)$ form a sequence of divided powers of $A$.

\footnotetext{
${ }^{2}$ For the reader who is mainly interested in the combinatorial aspects of the main results of this paper, the base field $K$ throughout this paper can be safely chosen to be the field $\mathbb{Q}$ of rational numbers.
} 
(c) One (hence also all) of $d(t), h(t)$ and $m(t)$ has all its coefficients primitive in $A$.

In the following remark, we would like to point out a connection of the notion of $\mathcal{N C S}$ systems with the notion of combinatorial Hopf algebras, which was first introduced by M. Aguiar, N. Bergeron and F. Sottile in [2].

Remark 2.3 First, as pointed out in Remark 2.17 in [28], when $A$ is a graded and connected Hopf algebra, and one of the statements of Proposition 2.2 holds, say statement (b). Furthermore assume in this case that the coefficients of $t^{m}(m \geq 0)$ of $g(t)$ are homogeneous with grading $m$. Then the data $(A, g(t))$ is equivalent to a combinatorial Hopf algebra structure on the graded dual Hopf algebra $A^{*}$ of $A$. For more details of the equivalence above, see Remark 2.17 in [28].

Since all other components of $\Omega$ are completely determined by $g(t)$ (see Lemma 2.5 of [28] or Theorem 2.6 below), the notion of $\mathcal{N C S}$ systems under the conditions above is also equivalent to the notion of combinatorial Hopf algebras. Therefore, from this point of view, the notion of $\mathcal{N C S}$ systems generalizes the notion of combinatorial Hopf algebras to associative $K$-algebras $A$, since, for $\mathcal{N} C S$ systems over $A, A$ does not have to be a bialgebra nor Hopf algebra and the equivalent conditions in Proposition 2.2 do not have to hold either.

On the other hand, we would also like to point out that the notion of $\mathcal{N}$ CS emphasizes the whole package of five generating functions of elements of $A$ instead of just one. In other words, it emphasizes solutions of the system of equations Eq. (2.1)(2.5). Even though, once one of the components of $\Omega$, say $g(t)$ again, is fixed, the other four will be given by the corresponding universal polynomials of NCSFs in coefficients of $g(t)$ (see Theorem 2.6 below), it is very often not trivial at all what values of these universal polynomials are, or in other words, it is still far away from clear how to write down directly and explicitly the other four components of $\Omega$.

The main aim of this paper is to construct explicitly a $\mathcal{N C S}$ system $\Omega_{\mathbb{T}}^{W}$ over the Grossman-Larson Hopf algebra $\mathcal{H}_{G L}^{W}$ of $W$-labeled trees without using any universal polynomials of NCSFs. Once we get the $\mathcal{N C S}$ system $\Omega_{\mathbb{T}}^{W}$ explicitly, then, by Theorem 2.6, these universal polynomials of NCSFs will be transformed into identities of coefficients of the corresponding components of $\Omega_{\mathbb{T}}^{W}$ (see Remark 4.8). Another immediate consequence is that we also get a very "visualizable representation", or more formally, a specialization of NCSFs by $W$-labeled rooted trees, which in return could be useful for studying and understanding certain properties of NCSFs.

Next, let us recall some of the NCSFs first introduced and studied in [10].

Let $\Lambda=\left\{\Lambda_{m} \mid m \geq 1\right\}$ be a sequence of noncommutative free variables and $\mathcal{N S y m}$ or $K\langle\Lambda\rangle$ the free associative algebra generated by $\Lambda$ over $K$. For convenience, we also set $\Lambda_{0}=1$. We denote by $\lambda(t)$ the generating function of $\Lambda_{m}(m \geq 0)$, i.e. we set

$$
\lambda(t):=\sum_{m \geq 0} t^{m} \Lambda_{m}=1+\sum_{k \geq 1} t^{m} \Lambda_{m} .
$$

In the theory of NCSFs [10], $\Lambda_{m}(m \geq 0)$ is the noncommutative analog of the $m^{t h}$ classical (commutative) elementary symmetric function and is called the $m^{t h}$ (noncommutative) elementary symmetric function. 
To define some other NCSFs, we consider Eqs. (2.2)-(2.5) over the free $K$-algebra $\mathcal{N} S y m$ with $f(t)=\lambda(t)$. The solutions for $g(t), d(t), h(t), m(t)$ exist and are unique, whose coefficients will be the NCSFs that we are going to define. Following the notation in [10] and [28], we denote the resulted 5-tuple by

$$
\Pi:=(\lambda(t), \sigma(t), \Phi(t), \psi(t), \xi(t))
$$

and write the last four generating functions of $\Pi$ explicitly as follows.

$$
\begin{aligned}
& \sigma(t)=\sum_{m \geq 0} t^{m} S_{m}, \\
& \Phi(t)=\sum_{m \geq 1} t^{m} \frac{\Phi_{m}}{m}, \\
& \psi(t)=\sum_{m \geq 1} t^{m-1} \Psi_{m}, \\
& \xi(t)=\sum_{m \geq 1} t^{m-1} \Xi_{m} .
\end{aligned}
$$

Now, for any $m \geq 1$, we define $S_{m}$ to be the $m^{\text {th }}$ (noncommutative) complete homogeneous symmetric function and $\Phi_{m}$ (resp. $\Psi_{m}$ ) the $m^{\text {th }}$ power sum symmetric function of the second (resp. first) kind. Note that, $\Xi_{m}(m \geq 1)$ were denoted by $\Psi_{m}^{*}$ in [10]. Due to Proposition 2.5 below, the NCSFs $\Xi_{m}(m \geq 1)$ do not play an important role in the NCSF theory (see the comments in page 234 in [10]). But, in the context of some other problems, relations of $\Xi_{m}$ 's with other NCSFs, especially, with $\Psi_{m}$ 's, are also important (see [30], for example). So here, following [28], we call $\Xi_{m} \in \mathcal{N} \operatorname{Sym}(m \geq 1)$ the $m^{\text {th }}$ (noncommutative) power sum symmetric function of the third kind.

The following two propositions proved in [10] and [16] will be very useful for our later arguments.

Proposition 2.4 For any unital commutative $\mathbb{Q}$-algebra $K$, the free algebra $\mathcal{N} S y m$ is freely generated by any one of the families of the NCSFs defined above.

Proposition 2.5 Let $\omega_{\Lambda}$ be the anti-involution of $\mathcal{N} S y m$ which fixes $\Lambda_{m}(m \geq 1)$. Then, for any $m \geq 1$, we have

$$
\begin{gathered}
\omega_{\Lambda}\left(S_{m}\right)=S_{m}, \\
\omega_{\Lambda}\left(\Phi_{m}\right)=\Phi_{m}, \\
\omega_{\Lambda}\left(\Psi_{m}\right)=\Xi_{m} .
\end{gathered}
$$

Next, let us recall the following graded $K$-Hopf algebra structure of $\mathcal{N} S y m$. It has been shown in [10] that $\mathcal{N} S y m$ is the universal enveloping algebra of the free Lie algebra generated by $\Psi_{m}(m \geq 1)$. Hence, it has a $K$-Hopf algebra structure as all 
other universal enveloping algebras of Lie algebras do. Its co-unit $\epsilon: \mathcal{N S}$ Sym $\rightarrow K$, co-product $\Delta$ and antipode $S$ are uniquely determined by

$$
\begin{aligned}
\epsilon\left(\Psi_{m}\right) & =0, \\
\Delta\left(\Psi_{m}\right) & =1 \otimes \Psi_{m}+\Psi_{m} \otimes 1, \\
S\left(\Psi_{m}\right) & =-\Psi_{m},
\end{aligned}
$$

for any $m \geq 1$.

Next, we introduce the weight of NCSFs by setting the weight of any monomial $\Lambda_{m_{1}}^{i_{1}} \Lambda_{m_{2}}^{i_{2}} \cdots \Lambda_{m_{k}}^{i_{k}}$ to be $\sum_{j=1}^{k} i_{j} m_{j}$. For any $m \geq 0$, we denote by $\mathcal{N} S y m_{[m]}$ the vector subspace of $\mathcal{N}$ Sym spanned by the monomials of $\Lambda$ of weight $m$. Then it is easy to see that

$$
\mathcal{N} \text { Sym }=\bigoplus_{m \geq 0} \mathcal{N S y m}_{[m]},
$$

which provides a grading for $\mathcal{N} S y m$.

Note that, it has been shown in [10], for any $m \geq 1$, the NCSFs $S_{m}, \Phi_{m}, \Psi_{m} \in$ $\mathcal{N S y m}_{[m]}$. By Proposition 2.5, this is also true for the NCSFs $\Xi_{m}$ 's. By the facts above and Eqs. (2.15)-(2.17), it is also easy to check that, with the grading given in Eq. (2.18), $\mathcal{N}$ Sym forms a graded $K$-Hopf algebra. Its graded dual is given by the space $\mathcal{Q} S y m$ of quasi-symmetric functions, which were first introduced by I. Gessel [11] (see [20] and [24] for more discussions).

Now we come back to our discussions on the $\mathcal{N} C S$ systems. From the definitions of the NCSFs above, we see that $(\mathcal{N} S y m, \Pi)$ obviously forms a $\mathcal{N} C S$ system. More importantly, as shown in Theorem 2.1 in [28], we have the following important theorem on the $\mathcal{N C S}$ system $(\mathcal{N}$ Sym, $\Pi)$.

Theorem 2.6 Let $A$ be a $K$-algebra and $\Omega$ a N CS system over A. Then,

(a) There exists a unique K-algebra homomorphism $\mathcal{S}: \mathcal{N} S y m \rightarrow A$ such that $\mathcal{S}^{\times 5}(\Pi)=\Omega$.

(b) If $A$ is further a $K$-bialgebra (resp. $K$-Hopf algebra) and one of the equivalent statements in Proposition 2.2 holds for the $\mathcal{N} C S$ system $\Omega$, then $\mathcal{S}: \mathcal{N}$ Sym $\rightarrow A$ is also a homomorphism of K-bialgebras (resp. K-Hopf algebras).

Remark 2.7 By applying the similar arguments as in the proof of Theorem 2.6, or simply taking the quotient over the two-sided ideal generated by the commutators of $\Lambda_{m}$ 's, it is easy to see that, over the category of commutative $K$-algebras, the universal $\mathcal{N C S}$ system is given by the generating functions of the corresponding classical (commutative) symmetric functions ([19]).

\section{The Grossman-Larson Hopf algebra and the Connes-Kreimer Hopf algebra}

Let $K$ be any unital commutative $\mathbb{Q}$-algebra and $W$ a non-empty subset of positive integers. In this section, we first fix some notations for unlabeled rooted trees and 
$W$-labeled rooted trees that will be used throughout this paper. We then recall the Connes-Kreimer Hopf algebra and the Grossman-Larson Hopf algebra of $W$-labeled forests and $W$-labeled rooted trees, respectively. Finally, by using the duality between the Grossman-Larson Hopf algebra and the Connes-Kreimer Hopf algebra (see Theorem 3.2), we prove a technic lemma, Lemma 3.4, that will play an important role in our later arguments.

First, let us fix the following notation which will be used throughout the rest of this paper.

Notation By a rooted tree we mean a finite 1-connected graph with one vertex designated as its root. For convenience, we also view the empty set $\emptyset$ as a rooted tree and call it the emptyset rooted tree. The rooted tree with a single vertex is called the singleton and denoted by $\circ$. There are natural ancestral relations between vertices. We say a vertex $w$ is a child of vertex $v$ if the two are connected by an edge and $w$ lies further from the root than $v$. In the same situation, we say $v$ is the parent of $w$. A vertex is called a leaf if it has no children.

Let $W \subseteq \mathbb{N}^{+}$be any non-empty subset of positive integers. A $W$-labeled rooted tree is a rooted tree with each vertex labeled by an element of $W$. If an element $m \in W$ is assigned to a vertex $v$, then $m$ is called the weight of the vertex $v$. When we speak of isomorphisms between unlabeled (resp. $W$-labeled) rooted trees, we will always mean isomorphisms which also preserve the root (resp. the root and also the labels of vertices). We will denote by $\mathbb{T}$ (resp. $\mathbb{T}^{W}$ ) the set of isomorphism classes of all unlabeled (resp. $W$-labeled) rooted trees. A disjoint union of any finitely many rooted trees (resp. $W$-labeled rooted trees) is called a rooted forest (resp. $W$-labeled rooted forest). We denote by $\mathbb{F}$ (resp. $\mathbb{F}^{W}$ ) the set of unlabeled (resp. $W$-labeled) rooted forests.

With these notions in mind, we establish the following notation.

(1) For any rooted tree $T \in \mathbb{T}^{W}$, we set the following notation:

- $\mathrm{rt}_{T}$ denotes the root vertex of $T$ and $O(T)$ the set of all the children of $\mathrm{rt}_{T}$. We set $o(T)=|O(T)|$ (the cardinal number of the set $O(T)$ ).

- $E(T)$ denotes the set of edges of $T$.

- $V(T)$ denotes the set of vertices of $T$ and $v(T)=|V(T)|$.

- $L(T)$ denotes the set of leaves of $T$ and $l(T)=|L(T)|$.

- For any $v \in V(T)$, we define the height of $v$ to be the number of edges in the (unique) geodesic connecting $v$ to $\mathrm{rt}_{T}$. The height of $T$ is defined to be the maximum of the heights of its vertices.

- For any $T \in \mathbb{T}^{W}$ and $T \neq \emptyset,|T|$ denotes the sum of the weights of all vertices of $T$. When $T=\emptyset$, we set $|T|=0$.

- For any $T \in \mathbb{T}^{W}$, we denote by $\operatorname{Aut}(T)$ the automorphism group of $T$ and $\alpha(T)$ the cardinal number of $\operatorname{Aut}(T)$.

(2) Any subset of $E(T)$ is called a cut of $T$. A cut $C \subseteq E(T)$ is said to be admissible if no two different edges of $C$ lie in the path connecting the root and a leaf. We denote by $\mathcal{C}(T)$ the set of all admissible cuts of $T$. Note that, the empty subset $\emptyset$ of $E(T)$ and $C=\{e\}$ for any $e \in E(T)$ are always admissible cuts. We will 
identify any edge $e \in E(T)$ with the admissible cut $C:=\{e\}$ and simply say the edge $e$ itself is an admissible cut of $T$.

(3) For any $T \in \mathbb{T}^{W}$ with $T \neq$ o, let $C \in \mathcal{C}(T)$ be an admissible cut of $T$ with $|C|=$ $m \geq 1$. Note that, after deleting the edges in $C$ from $T$, we get a disjoint union of $m+1$ rooted trees, say $T_{0}, T_{1}, \ldots, T_{m}$ with $\operatorname{rt}(T) \in V\left(T_{0}\right)$. We define $R_{C}(T)=$ $T_{0} \in \mathbb{T}^{W}$ and $P_{C}(T) \in \mathbb{F}^{W}$ the rooted forest formed by $T_{1}, \ldots, T_{m}$.

(4) For any disjoint admissible cuts $C_{1}$ and $C_{2}$, we say " $C_{1}$ lies above $C_{2}$ ", and write $C_{1} \succ C_{2}$, if $C_{2} \subseteq E\left(R_{C_{1}}(T)\right)$. This merely says that all edges of $C_{2}$ remain when we remove all edges of $C_{1}$ and $P_{C_{1}}(T)$. Note that this relation is not transitive. When we write $C_{1} \succ \cdots \succ C_{r}$ for $C_{1}, \ldots, C_{r} \in \mathcal{C}(T)$, we will mean that $C_{i} \succ C_{j}$ whenever $i<j$.

(5) For any $T \in \mathbb{T}^{W}$, we say $T$ is a chain if its underlying rooted tree is a rooted tree with a single leaf. We say $T$ is a shrub if its underlying rooted tree is a rooted tree of height 1 . We say $T$ is primitive if its root has only one child. For any $m \geq 1$, we set $\mathbb{H}_{m}, \mathbb{S}_{m}$ and $\mathbb{P}_{m}$ to be the sets of the chains, shrubs and primitive rooted trees $T$ of weight $|T|=m$, respectively. $\mathbb{H}, \mathbb{S}$ and $\mathbb{P}$ are set to be the unions of $\mathbb{H}_{m}, \mathbb{S}_{m}$ and $\mathbb{P}_{m}$, respectively, for all $m \geq 1$. have

For example, in the case where $W=\{1\}$, which allows not to write the labels, we

$$
\begin{aligned}
& \mathbb{H}=\{.,:, \vdots, \vdots, \ldots\}, \\
& \mathbb{S}=\{:, \ddot{\vee}, \dddot{\vee}, \underset{\vee}{ } \ldots\},
\end{aligned}
$$

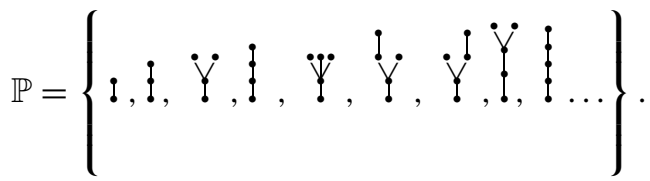

For any non-empty $W \subseteq \mathbb{N}^{+}$, we define the following operations for $W$-labeled rooted forests. For any $F \in \mathbb{F}^{W}$ which is disjoint union of $W$-labeled rooted trees $T_{i}$ $(1 \leq i \leq m)$, we set $B_{+}\left(T_{1}, T_{2}, \cdots, T_{m}\right)$ the rooted tree obtained by connecting roots of $T_{i}(1 \leq i \leq m)$ to a newly added root. We will keep the labels for the vertices of $B_{+}\left(T_{1}, T_{2}, \cdots, T_{m}\right)$ from $T_{i}$ 's, but for the root, we label it by 0 . For convenience, we also fix the following short convention for the operation $B_{+}$. For the empty rooted tree $\emptyset$, we set $B_{+}(\varnothing)$ to be the singleton labeled by 0 . For any $T_{i} \in \mathbb{T}^{W}(1 \leq i \leq m)$ and $j_{i} \geq 1$, the notation $B_{+}\left(T_{1}^{j_{1}}, T_{2}^{j_{2}}, \cdots, T_{m}^{j_{m}}\right)$ denotes the rooted tree obtained by applying the operation $B_{+}$to $j_{1}$-copies of $T_{1} ; j_{2}$-copies of $T_{2}$; and so on. Later, for any unital $\mathbb{Q}$-algebra $K$ and $m \geq 1$, we will also extend the operation $B_{+}$multilinearly to a linear map $B_{+}$from $\left(\mathcal{H}_{C K}^{W}\right)^{\times m}$ to $\mathcal{H}_{G L}^{W}$, where $\mathcal{H}_{C K}^{W}$ and $\mathcal{H}_{G L}^{W}$ at this moment are the vector spaces spanned over $K$ by the elements of $\mathbb{T}^{W}$ and $B_{+}\left(\mathbb{T}^{W}\right)$, respectively.

Now, we set $\overline{\mathbb{T}}^{W}:=\left\{B_{+}(F) \mid F \in \mathbb{F}^{W}\right\}$. Then, $B_{+}: \mathbb{F}^{W} \rightarrow \overline{\mathbb{T}}^{W}$ becomes a bijection. We denote by $B_{-}: \overline{\mathbb{T}}^{W} \rightarrow \mathbb{F}^{W}$ the inverse map of $B_{+}$. More precisely, for any 
$T \in \overline{\mathbb{T}}^{W}, B_{-}(T)$ is the $W$-labeled rooted forest obtained by cutting off the root of $T$ as well as all edges connecting to the root in $T$.

Note that, precisely speaking, elements of $\overline{\mathbb{T}}^{W}$ are not $W$-labeled trees for $0 \notin W$. But, if we set $\bar{W}=W \cup\{0\}$, then we can view $\overline{\mathbb{T}}^{W}$ as a subset of $\bar{W}$-labeled rooted trees $T$ with the root $\mathrm{rt}_{T}$ labeled by 0 and all other vertices labeled by non-zero elements of $\bar{W}$. We extend the definition of the weight for elements of $\mathbb{F}^{W}$ to elements of $\overline{\mathbb{T}}^{W}$ by simply counting the weight of roots by zero. We set $\overline{\mathbb{S}}_{m}^{W}:=B_{+}\left(\mathbb{S}_{m}^{W}\right)(m \geq$ 1) and $\overline{\mathbb{S}}^{W}:=B_{+}\left(\mathbb{S}^{W}\right)$. We also define $\overline{\mathbb{H}}_{m}^{W}, \overline{\mathbb{P}}_{m}^{W}, \overline{\mathbb{H}}^{W}$ and $\overline{\mathbb{P}}^{W}$ in the similar way.

Next we fix a unital commutative $\mathbb{Q}$-algebra $K$ and a non-empty subset of positive integers $W$, and first recall the Connes-Kreimer Hopf algebras $\mathcal{H}_{C K}^{W}$ of $W$-labeled rooted forests.

As a $K$-algebra, the Connes-Kreimer Hopf algebra $\mathcal{H}_{C K}^{W}$ is the free commutative algebra generated by formal variables $\left\{X_{T} \mid T \in \mathbb{T}^{W}\right\}$. Here, for convenience, we will still use $T$ to denote the variable $X_{T}$ in $\mathcal{H}_{C K}^{W}$. The $K$-algebra product is given by the disjoint union. The identity element of this algebra, denoted by 1 , is the free variable $X_{\emptyset}$ corresponding to the emptyset rooted tree $\emptyset$. The coproduct $\Delta: \mathcal{H}_{C K}^{W} \rightarrow$ $\mathcal{H}_{C K}^{W} \otimes \mathcal{H}_{C K}^{W}$ is uniquely determined by setting

$$
\begin{aligned}
& \Delta(1)=1 \otimes 1, \\
& \Delta(T)=T \otimes 1+\sum_{C \in \mathcal{C}(T)} P_{C}(T) \otimes R_{C}(T) .
\end{aligned}
$$

The co-unit $\epsilon: \mathcal{H}_{C K}^{W} \rightarrow K$ is the $K$-algebra homomorphism which sends $1 \in \mathcal{H}_{C K}^{W}$ to $1 \in K$ and $T$ to 0 for any $T \in \mathbb{T}^{W}$ with $T \neq \emptyset$. With the operations defined above and the grading given by the weight, the vector space $\mathcal{H}_{C K}^{W}$ forms a connected graded commutative bialgebra. Since any connected graded bialgebra is a Hopf algebra, there is a unique antipode $S: \mathcal{H}_{C K}^{W} \rightarrow \mathcal{H}_{C K}^{W}$ that makes $\mathcal{H}_{C K}^{W}$ a connected graded commutative $K$-Hopf algebra. For a formula for the antipode, see [8, 9].

Next we recall the Grossman-Larson Hopf algebra of labeled rooted trees. As a vector space, the Grossman-Larson Hopf algebra $\mathcal{H}_{G L}^{W}$ is the vector space spanned by elements of $\overline{\mathbb{T}}^{W}$ over $K$. For any $T \in \overline{\mathbb{T}}^{W}$, we will still denote by $T$ the vector in $\mathcal{H}_{G L}^{W}$ that is corresponding to $T$. The algebra product is defined as follows.

For any $T, S \in \overline{\mathbb{T}}^{W}$ with $T=B_{+}\left(T_{1}, T_{2}, \cdots, T_{m}\right)$, we set $T \cdot S$ to be the sum of the rooted trees obtained by connecting the roots of $T_{i}(1 \leq i \leq m)$ to vertices of $S$ in all possible $m^{v(S)}$ different ways. Note that, the identity element with respect to this algebra product is given by the singleton $\circ=B_{+}(\emptyset)$. But we will denote it by 1 .

To define the co-product $\Delta: \mathcal{H}_{G L}^{W} \rightarrow \mathcal{H}_{G L}^{W} \otimes \mathcal{H}_{G L}^{W}$, we first set

$$
\Delta(\circ)=\circ \otimes \circ
$$

Now let $T \in \overline{\mathbb{T}}^{W}$ with $T \neq \circ$, say $T=B_{+}\left(T_{1}, T_{2}, \cdots, T_{m}\right)$ with $m \geq 1$ and $T_{i} \in$ $\mathbb{T}^{W}(1 \leq i \leq m)$. For any non-empty subset $I \subseteq\{1,2, \cdots, m\}$, we denote by $B_{+}\left(T_{I}\right)$ the rooted tree obtained by applying the $B_{+}$operation to the rooted trees $T_{i}$ with $i \in I$. For convenience, when $I=\emptyset$, we set $B_{+}\left(T_{I}\right)=1$. With this notation fixed, the 
co-product for $T$ is given by

$$
\Delta(T)=\sum_{I \sqcup J=\{1,2, \cdots, m\}} B_{+}\left(T_{I}\right) \otimes B_{+}\left(T_{J}\right) .
$$

The co-unit $\epsilon: \mathcal{H}_{G L}^{W} \rightarrow K$ is the $K$-algebra homomorphism which sends $1 \in \mathcal{H}_{G L}^{W}$ to $1 \in K$ and $T$ to 0 for any $T \in \overline{\mathbb{T}}^{W}$ with $T \neq \emptyset$. With the operations defined above and the grading given by the weight, the vector space $\mathcal{H}_{G L}^{W}$ forms a graded commutative bialgebra. Therefore, there is a unique antipode $S: \mathcal{H}_{C K}^{W} \rightarrow \mathcal{H}_{C K}^{W}$ that makes $\mathcal{H}_{C K}^{W}$ a graded $K$-Hopf algebra. Actually, by the general recurrent formula, we can write down the antipode of $\mathcal{H}_{G L}^{W}$ as follows.

Note that the singleton $\circ$ is a group-like element and $S(\circ)=\circ^{-1}=\circ$. Now assume $T \neq \circ$ and write $T=B_{+}\left(T_{1}, T_{2}, \cdots, T_{m}\right)$ with $m \geq 1$ and $T_{i} \in \mathbb{T}^{W}$. Let $I:=\{1,2, \cdots, m\}$. For $1 \leq r \leq m$, let $\mathcal{P}_{r}$ be the set of $r$-tuples $\left(I_{1}, I_{2}, \cdots, I_{r}\right)$ of disjoint non-empty subsets of $I$ whose union is $I$. In other words, $\mathcal{P}_{r}$ is the set of all ordered partitions of $I$ into $r$ non-empty subsets of $I$.

Lemma 3.1 Let $S$ denote the antipode of the Grossman-Larson Hopf algebra $\mathcal{H}_{G L}^{W}$ of $W$-labeled rooted trees. Then, for any $T \in \overline{\mathbb{T}}^{W}$ with $T \neq 0$ as above, we have

$$
S(T)=\sum_{r=1}^{m}(-1)^{r} \sum_{\left(I_{1}, \cdots, I_{r}\right) \in \mathcal{P}_{r}} B_{+}\left(T_{I_{1}}\right) B_{+}\left(T_{I_{2}}\right) \cdots B_{+}\left(T_{I_{r}}\right)
$$

Proof By the general recurrent formula for the antipode of connected cocommutative graded Hopf algebras, we know that the antipode $S$ of $\mathcal{H}_{G L}^{W}$ satisfies the following equation:

$$
S(T)=-T-\sum_{(I, J) \in \mathcal{P}_{2}} S\left(B_{+}\left(T_{I}\right)\right) B_{+}\left(T_{J}\right)
$$

Then it is easy to check directly that, for any $T \in \overline{\mathbb{T}}^{W}, S(T)$ given by Eq. (3.5) does satisfy Eq. (3.6). Since the solution to Eq. (3.6) is unique, the antipode $S$ of $\mathcal{H}_{G L}^{W}$ is actually given by Eq. (3.5).

Note that, from Eq. (3.4), it is easy to see that, a rooted tree $T \in \overline{\mathbb{T}}^{W}$ is a primitive element of the Hopf algebra $\mathcal{H}_{G L}^{W}$ iff it is a primitive rooted tree in the sense that we defined before, namely the root of $T$ has one and only one child. It is noticeable that the set of primitive $W$-labeled rooted trees is a basis of the space $\operatorname{Prim}\left(\mathcal{H}_{G L}^{W}\right)$ of primitive elements of $\mathcal{H}_{G L}^{W}$. Moreover, by the Milnor-Moore theorem, $\mathcal{H}_{G L}^{W}$ is isomorphic to $\mathcal{U}\left(\operatorname{Prim}\left(\mathcal{H}_{G L}^{W}\right)\right)$.

The relation between the Grossman-Larson Hopf algebra $\mathcal{H}_{G L}^{W}$ and the ConnesKreimer Hopf algebra $\mathcal{H}_{C K}^{W}$ is given by the following important theorem, which was proved in [13] and [8,9]. 
Theorem 3.2 The Hopf algebras $\mathcal{H}_{G L}^{W}$ and $\mathcal{H}_{C K}^{W}$ are graded dual to each other. The pairing is given by, for any $T \in \overline{\mathbb{T}}^{W}$ and $S \in \mathbb{F}^{W}$,

$$
\langle T, F\rangle= \begin{cases}0, & \text { if } T \not B_{+}(F), \\ \alpha(T), & \text { if } T \simeq B_{+}(F) .\end{cases}
$$

Furthermore, the following theorem on the algebra structure constants of $\mathcal{H}_{G L}^{W}$ was also proved in [13] and [8,9].

Theorem 3.3 For any $T^{\prime}, S \in \overline{\mathbb{T}}^{W}$, We have

$$
T^{\prime} \cdot S=\sum_{T \in \overline{\mathbb{T}}^{W}} \sum_{\substack{C \in \mathcal{C}(T) \\ B_{+}\left(P_{C}(T)\right) \sim T^{\prime}, R_{C}(T) \sim S .}} \frac{\alpha\left(T^{\prime}\right) \alpha(S)}{\alpha(T)} T .
$$

Note that, Eq. (3.8) suggests that it is much more convenient to work with the basis $\left\{\mathcal{V}_{T}:=T / \alpha(T) \mid T \in \overline{\mathbb{T}}^{W}\right\}$ than the basis $\left\{T \mid T \in \overline{\mathbb{T}}^{W}\right\}$. For example, in terms of $\mathcal{V}_{T}$, Eq. (3.8) becomes

$$
\mathcal{V}_{T^{\prime}} \cdot \mathcal{V}_{S}=\sum_{T \in \overline{\mathbb{T}}^{W}} \sum_{\substack{C \in \mathcal{C}(T) \\ B_{+}\left(P_{C}(T)\right) \sim T^{\prime} \\ R_{C}(T) \sim S .}} \mathcal{V}_{T}
$$

Finally, we extend Theorem 3.3 to a more general setting (see Lemma 3.4 below). It can be viewed as a generalization of Lemma 2.8 in [26] which essentially is the case of Lemma 3.4 when only primitive rooted trees are involved. First, let us fix the following notation.

Let $\vec{C}=\left(C_{1}, \ldots, C_{r}\right) \in \mathcal{C}(T)^{\times r}$ be a sequence of admissible cuts with $C_{1} \succ$ $\cdots \succ C_{r}$. We define a sequence of $T_{\vec{C}, 1}, \ldots, T_{\vec{C}, r+1} \in \overline{\mathbb{T}}^{W}$ as follows: we first set $T_{\vec{C}, 1}=B_{+}\left(P_{C_{1}}(T)\right)$ and let $S_{1}=R_{C_{1}}(T)$. Note that $C_{2}, \ldots, C_{r} \in \mathcal{C}\left(S_{1}\right)$. We then set $T_{\vec{C}, 2}=B_{+}\left(P_{C_{2}}\left(S_{1}\right)\right)$ and $S_{2}=R_{C_{2}}\left(S_{1}\right)$ and repeat this procedure until we get $S_{r}=R_{C_{r}}\left(S_{r-1}\right)$ and then set $T_{\vec{C}, r+1}=S_{r}$. In the case that, each $C_{i}(1 \leq i \leq r)$ consists of a single edge, say $e_{i} \in E(T)$, we simply denote $T_{\vec{C}, i}$ by $T_{e_{i}}$.

Now we fix a positive integer $r$ and let $y=\left\{y_{T}^{(i)} \mid 1 \leq i \leq r ; T \in \overline{\mathbb{T}}^{W}\right\}$ be a collection of commutative formal variables.

Lemma 3.4 For any $r \geq 1$, we have,

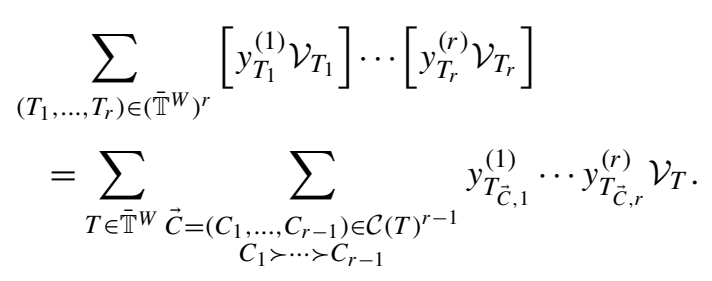


Proof We denote the LHS of Eq. (3.10) by $Q$ and write it as

$$
Q=\sum_{T \in \overline{\mathbb{T}}^{W}} y_{T} \mathcal{V}_{T}
$$

Then, by Eq. (3.7) $y_{T}=\left\langle Q, B_{-}(T)\right\rangle$ for any $T \in \overline{\mathbb{T}}^{W}$, where $B_{-}(T)$ is the forest obtained by deleting the root of $T$. So:

$$
\begin{aligned}
y_{T} & =\sum_{\left(T_{1}, \ldots, T_{r}\right)} y_{T_{1}}^{(1)} \cdots y_{T_{r}}^{(r)}\left\langle\mathcal{V}_{T_{1}} \ldots \mathcal{V}_{T_{r}}, B_{-}(T)\right\rangle \\
& =\sum_{\left(T_{1}, \ldots, T_{r}\right)} y_{T_{1}}^{(1)} \cdots y_{T_{r}}^{(r)}\left\langle\mathcal{V}_{T_{1}} \otimes\left(\mathcal{V}_{T_{2}} \cdots \mathcal{V}_{T_{r}}\right), \Delta\left(B_{-}(T)\right)\right\rangle \\
& =\sum_{\left(T_{1}, \ldots, T_{r}\right)} y_{T_{1}}^{(1)} \cdots y_{T_{r}}^{(r)}\left\langle\mathcal{V}_{T_{1}} \otimes \mathcal{V}_{T_{2}} \otimes\left(\mathcal{V}_{T_{3}} \cdots \mathcal{V}_{T_{r}}\right),(I \otimes \Delta) \circ \Delta\left(B_{-}(T)\right)\right\rangle,
\end{aligned}
$$

where $I$ is the identity map of $\mathcal{H}_{C K}^{W}$. Repeating the process above:

$$
=\sum_{\left(T_{1}, \ldots, T_{r}\right)} y_{T_{1}}^{(1)} \cdots y_{T_{r}}^{(r)}\left\langle\mathcal{V}_{T_{1}} \otimes \cdots \otimes \mathcal{V}_{T_{r}},\left(I^{\otimes(r-2)} \otimes \Delta\right) \circ \cdots \circ \Delta\left(B_{-}(T)\right)\right\rangle .
$$

One the other hand, by definition of the coproduct of $\mathcal{H}_{C K}^{W}$ and definition of $\succ$, we have

$$
\begin{aligned}
& \left(I^{\otimes(r-2)} \otimes \Delta\right) \circ \cdots \circ(I \otimes \Delta) \circ \Delta\left(B_{-}(T)\right) \\
& \quad=\sum_{\substack{\vec{C}=\left(C_{1}, \ldots, C_{r-1}\right) \in \mathcal{C}(T)^{r-1} \\
C_{1} \succ \cdots \succ C_{r-1}}} B_{-}\left(T_{\vec{C}, 1}\right) \otimes \ldots \otimes B_{-}\left(T_{\vec{C}, r}\right) .
\end{aligned}
$$

Therefore, we get

$$
y_{T}=\sum_{\substack{\vec{C}=\left(C_{1}, \ldots, C_{r-1} \in \mathcal{C}(T)^{r-1} \\ C_{1} \succ \cdots \succ C_{r-1}\right.}} y_{T_{\vec{C}, 1}^{(1)} \cdots y_{T_{\vec{C}, r}}^{(r)}} .
$$

\section{A $\mathcal{N}$ CS system over the Grossman-Larson Hopf algebra $\mathcal{H}_{G L}^{W}$ of $W$-labeled rooted trees}

In this section, for any non-empty $W \subseteq \mathbb{N}^{+}$, we construct a $\mathcal{N C S}$ system $\Omega_{\mathbb{T}}^{W}$ over the Grossman-Larson Hopf algebra $\mathcal{H}_{G L}^{W}$. First, let us introduce the following generating functions of certain elements of $\mathcal{H}_{G L}^{W}$, which will be the components of the $\mathcal{N}$ CS system $\Omega_{\mathbb{T}}^{W}$ corresponding to $f(t), g(t), h(t)$ and $m(t)$ according the notation in 
Definition 2.1.

$$
\begin{aligned}
& \tilde{f}(t):=\sum_{T \in \overline{\mathbb{S}}^{W}}(-1)^{o(T)+|T|} t^{|T|} \mathcal{V}_{T}=1+\sum_{\substack{T \in \overline{\mathbb{S}}^{T} W \\
T \neq \circ}}(-1)^{o(T)+|T|} t^{|T|} \mathcal{V}_{T} \\
& \tilde{g}(t):=\sum_{T \in \overline{\mathbb{T}}^{W}} t^{|T|} \mathcal{V}_{T}=1+\sum_{\substack{T \in \overline{\mathbb{T}}^{W} W \\
T \neq 0}} t^{|T|} \mathcal{V}_{T}, \\
& \tilde{h}(t):=\sum_{T \in \overline{\mathbb{H}}^{W}} t^{|T|-1} \beta_{T} \mathcal{V}_{T} \\
& \tilde{m}(t):=\sum_{T \in \overline{\mathbb{P}}^{W}} t^{|T|-1} \gamma_{T} \mathcal{V}_{T}
\end{aligned}
$$

where, for any $T \in \overline{\mathbb{H}}^{W}$ (resp. $T \in \overline{\mathbb{P}}^{W}$ ), $\beta_{T}$ (resp. $\gamma_{T}$ ) is the weight of the unique leaf (resp. the unique child of the root) of $T$. Note that, for the singleton $T=\circ$, we have $\beta_{T}=\gamma_{T}=0$. So $\tilde{h}(0)=\tilde{m}(0)=0$.

For example, when $W=\{1,2\}$, we have

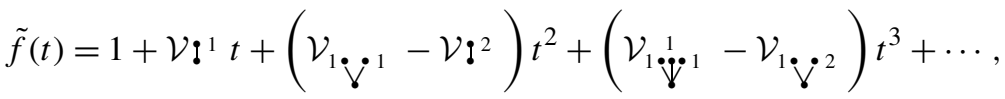

$$
\begin{aligned}
& \tilde{g}(t)=1+\mathcal{V} !^{1} t+\left(\mathcal{V}:^{2}+\mathcal{V}_{1} \ddot{\vartheta}^{1}+\mathcal{V}_{!_{1}^{1}}\right) t^{2}
\end{aligned}
$$

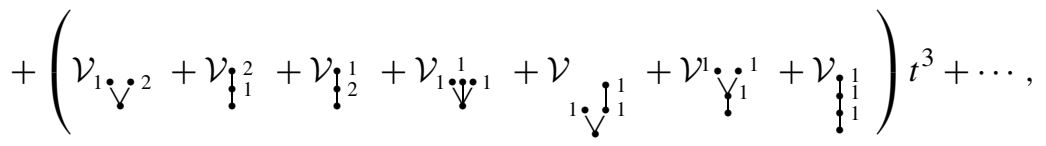

$$
\begin{aligned}
& \tilde{h}(t)=\mathcal{V}_{\mathfrak{l}^{1}}+\left(\mathcal{V}_{!_{1}^{1}}+2 \mathcal{V} !^{2}\right) t+\left(\mathcal{V}_{!_{1}^{1}}+2 \mathcal{V}_{!_{1}^{2}}+\mathcal{V}_{!_{2}^{1}}\right) t^{2}+\cdots, \\
& \tilde{m}(t)=\mathcal{V} !^{1}+\left(\mathcal{V} !_{1}^{1}+2 \mathcal{V} !^{2}\right) t+\left(\mathcal{V}_{!_{1}^{2}}+2 \mathcal{V}_{!_{2}^{1}}^{1}+\mathcal{V}^{1} \ddot{!}_{1}^{1}+\mathcal{V}_{\vdots_{1}^{1}}\right) t^{2}+\cdots
\end{aligned}
$$

Note that, from Eq. (4.1), we have

$$
\tilde{f}(-t)=\sum_{T \in \overline{\mathbb{S}} W}(-1)^{o(T)} t^{|T|} \mathcal{V}_{T}=1+\sum_{\substack{T \in \overline{\mathbb{S}} W \\ T \neq \circ}}(-1)^{o(T)} t^{|T|} \mathcal{V}_{T}
$$

A different way to look at the generating function $\tilde{f}(-t)$ is as follows.

For any $m \in W$, let $\kappa_{m}$ denote the singleton labeled by $m$ and set

$$
\kappa(t):=\sum_{m \in W} t^{m} \kappa_{m}
$$




\section{Lemma 4.1}

$$
\tilde{f}(-t)=1+\sum_{d \geq 1} \frac{(-1)^{d}}{d !} B_{+}\left(\kappa(t)^{d}\right)
$$

where $B_{+}\left(\kappa(t)^{d}\right)$ denotes the element obtained by applying $B_{+}$to $d$-copies of $\kappa(t)$.

Proof First, it is easy to see that, the only terms that can appear in the expansion of the RHS of Eq. (4.7) are shrubs. Secondly, from the definition of the operation $B_{+}$, we see that $B_{+}$is symmetric and multi-linear in its components. Therefore, we can expand the term $B_{+}\left(\kappa(t)^{d}\right)$ into a linear combination of rooted trees $S \in \overline{\mathbb{T}}^{W}$ in a similar way as we expand the power $\left(\sum_{m \in W} t^{m} u_{m}\right)^{d}$ for some free commutative variables $u_{m}(m \in W)$.

Now, for any shrub $S \in \overline{\mathbb{S}}^{W}$ with $S \neq$ o, let $\left\{m_{j} \in W \mid 1 \leq j \leq N\right\}$ be the set of all labels of the leaves of $S$. Let $i_{j} \geq 1(1 \leq j \leq N)$ be the number of the $m_{j}$-labeled leaves of $S$. Then we have

$$
\begin{aligned}
o(S) & =\sum_{1 \leq j \leq N} i_{j}, \\
|S| & =\sum_{1 \leq j \leq N} i_{j} m_{j}, \\
\alpha(S) & =\prod_{1 \leq j \leq N}\left(i_{j}\right) !
\end{aligned}
$$

Now let us consider the coefficient $c_{S}$ of $S$ in the linear expansion of the RHS of Eq. (4.7). By the observations in the first paragraph of the proof and Eqs. (4.8)-(4.10), it is easy to see that we have

$$
\begin{aligned}
c_{S} & =\frac{(-1)^{o(S)} t^{|S|}}{o(S) !}\left(\begin{array}{c}
o(S) \\
i_{1}, \cdots, i_{N}
\end{array}\right) \\
& =(-1)^{o(S)} \frac{t^{|S|}}{\prod_{1 \leq j \leq N}\left(i_{j}\right) !} \\
& =(-1)^{o(S)} \frac{t^{|S|}}{\alpha(S)},
\end{aligned}
$$

which is same as the coefficient of $S$ in $\tilde{f}(-t)$ since $\mathcal{V}_{S}=\frac{1}{\alpha(S)} S$. Hence we are done.

To define the generating function $\tilde{d}(t)$ for the third component of the underconstruction $\mathcal{N C S}$ system $\Omega_{\mathbb{T}}^{W}$, we first need the following definition.

\section{Definition 4.2}

(a) We define a constant $\theta_{T} \in \mathbb{Q}$ for each unlabeled rooted tree $T$ as follows. 
(1) For the singleton $\circ$ and any non-primitive rooted tree $T \in \mathbb{T}$, i.e. $o(T)>1$, we set $\theta_{\circ}=\theta_{T}=0$.

(2) For $T=B_{+}$(o), we set $\theta_{T}=1$.

(3) For any primitive $T \in \mathbb{P}$ with $v(T) \geq 3$, we define $\theta_{T}$ inductively by

$$
\theta_{T}=1-\sum_{m \geq 2} \frac{1}{m !} \sum_{\substack{\vec{e}=\left(e_{1}, \ldots, e_{m-1} \in \in E(T)^{m-1} \\ e_{1} \succ \cdots \succ e_{m-1}\right.}} \theta_{T_{e_{1}}} \theta_{T_{e_{2}}} \cdots \theta_{T_{e_{m}}},
$$

where $T_{e_{i}}$ 's in the equation above have been defined before Lemma 3.4.

(b) For any $W \subseteq \mathbb{N}$ and $W$-labeled rooted tree $T$, we set $\theta_{T}:=\theta_{\bar{T}}$, where $\bar{T}$ is the underlying unlabeled rooted tree of $T$.

Remark 4.3 As we will show later in Sect. 5, the constant $\theta_{T}(T \in \mathbb{T})$ has a natural combinatorial interpretations as follows. If we write $T=B_{+}(F)$ for some rooted forest $F \in \mathbb{F}$ and let $\Omega(F, s)$ be the order polynomial (see [23]) of $F$, then $\theta_{T}$ will be the coefficient of $s$ of $\Omega(F, s)$ (see Proposition 5.8 in Sect. 5). Furthermore, if we denote by $\nabla: K[s] \rightarrow K[s]$ the linear operator which maps any $f(s) \in K[s]$ to $f(s)-f(s-1)$, then $\theta_{T}$ is also the coefficient of $s$ of the polynomial $\nabla \Omega(T, s)$ (see Corollary 5.9). In order to keep our on-going arguments more focus, we will postpone to Sect. 5 a detailed discussion on these combinatorial interpretations of $\theta_{T}$ $(T \in \mathbb{T})$.

Example 4.4 By Eqs. (5.3), (5.5), (5.10) and (5.13) in Sect. 5, it is easy to check that, for the chains $C_{m}$ 's and $B_{+}\left(S_{m}\right)$ of the shrubs $S_{m}$ 's, we have

$$
\begin{aligned}
\theta_{C_{m}} & =\frac{1}{m-1} \quad \text { for any } m \geq 2 . \\
\theta_{B_{+}\left(S_{m}\right)} & =(-1)^{m} b_{m} \quad \text { for any } m \geq 0,
\end{aligned}
$$

where $b_{m}(m \geq 0)$ are the Bernoulli numbers which are defined by the generating function

$$
\frac{x}{e^{x}-1}=\sum_{m=0}^{\infty} b_{m} \frac{x^{m}}{m !} .
$$

Now, we introduce the following generating function:

$$
\tilde{d}(t):=\sum_{T \in \overline{\mathbb{P}}^{W}} t^{|T|} \theta_{T} \mathcal{V}_{T}
$$

For example, when $W=\{1,2\}$, we have

$$
\begin{aligned}
\tilde{d}(t)= & \mathcal{V} \mathfrak{!}^{1} t+\left(\frac{1}{2} \mathcal{V}_{!_{1}^{1}}+\mathcal{V} !^{2}\right) t^{2} \\
& +\left(\frac{1}{2} \mathcal{V}_{!_{1}^{2}}+\frac{1}{2} \mathcal{V} !_{{ }^{2}}+\frac{1}{6} \mathcal{V}^{1} \ddot{\varliminf}^{1}+\frac{1}{3} \mathcal{V}_{\vdots_{1}^{1}}\right) t^{3}+\cdots
\end{aligned}
$$


Set

$$
\Omega_{\mathbb{T}}^{W}:=(\tilde{f}(t), \tilde{g}(t), \tilde{d}(t), \tilde{h}(t), \tilde{m}(t)) .
$$

Then, the main result of this section is the following theorem.

Theorem 4.5 For any non-empty set $W \subseteq \mathbb{N}, \Omega_{\mathbb{T}}^{W}$ forms a $\mathcal{N} C S$ system over the Grossman-Larson Hopf algebra $\mathcal{H}_{G L}^{W}$.

Proof Note that, by Eqs. (4.1) and (4.2), we have $\tilde{f}(0)=\tilde{g}(0)=1$, hence it will be enough to show Eqs. (2.2)-(2.5) in Definition 2.1 are satisfied by the generating functions in $\Omega_{\mathbb{T}}^{W}$.

Let us start with Eq. (2.2). First, note that, since $\tilde{g}(0)=1, \tilde{g}(t)$ as an element of $\mathcal{H}_{G L}^{W}[[t]]$ does have both left and right inverses. So we only need show $\tilde{f}(-t) \tilde{g}(t)=1$ for $\tilde{g}(t) \tilde{f}(-t)=1$ will follow automatically. Secondly, by Eq. (4.5) and Lemma 3.4 with $y_{T}^{(1)}=(-1)^{o(T)} t^{|T|}$ if $T \in \overline{\mathbb{S}}^{W}$ and 0 otherwise, and $y_{T}^{(2)}=t^{|T|}$ for any $T \in \overline{\mathbb{T}}^{W}$, we have

$$
\begin{aligned}
& \tilde{f}(-t) \tilde{g}(t)=\left(\sum_{T^{\prime} \in \overline{\mathbb{S}} W}(-1)^{o\left(T^{\prime}\right)} t^{\left|T^{\prime}\right|} \mathcal{V}_{T^{\prime}}\right)\left(\sum_{T^{\prime \prime} \in \overline{\mathbb{T}}^{W}} t^{\left|T^{\prime \prime}\right|} \mathcal{V}_{T^{\prime \prime}}\right) \\
& =\sum_{T \in \overline{\mathbb{T}}^{W}} t^{|T|}\left(\sum_{\substack{C \in \mathcal{C}(T) \\
B_{+}\left(P_{C}(T)\right) \in \overline{\mathbb{S}}^{W}}}(-1)^{o\left(B_{+}\left(P_{C}(T)\right)\right)}\right) \mathcal{V}_{T} .
\end{aligned}
$$

First, note that, for any rooted tree $T$ and an admissible cut $C$ of $T, B_{+}\left(P_{C}(T)\right)$ is a shrub iff each edge in $C$ is the unique edge connecting with a leaf of $T$. Therefore, the set of all admissible cuts $C$ such that $B_{+}\left(P_{C}(T)\right) \in \overline{\mathbb{S}}^{W}$ is in 1-1 correspondence with the set of subsets of leaves of $T$. Secondly, when $B_{+}\left(P_{C}(T)\right) \in \overline{\mathbb{S}}^{W}$ for an admissible cut $C, o\left(B_{+}\left(P_{C}(T)\right)\right)$ is same as the cardinal number $|C|$ of the cut $C$. With these observations, for any $T \in \overline{\mathbb{T}}^{W}$ with $l(T):=|L(T)|>0$, we have

$$
\sum_{\substack{C \in \mathcal{C}(T) \\
B_{+}\left(P_{C}(T)\right) \in \overline{\mathbb{S}}^{W}}}(-1)^{|C|}=\sum_{k=0}^{l(T)}(-1)^{k}\left(\begin{array}{c}
l(T) \\
k
\end{array}\right)=0 .
$$

Combining Eqs. (4.17) and (4.18), we get $\tilde{f}(-t) \tilde{g}(t)=1$ and hence Eq. (2.2) for the system $\Omega_{\mathbb{T}}^{W}$.

Now, let us prove Eq. (2.3) as follows.

$$
\begin{aligned}
e^{\tilde{d}(t)} & =\sum_{k \geq 0} \frac{1}{k !} \tilde{d}(t)^{k} \\
& =1+\sum_{k \geq 1} \frac{1}{k !}\left(\sum_{T \in \overline{\mathbb{P}}^{W}} t^{|T|} \theta_{T} \mathcal{V}_{T}\right)^{k}
\end{aligned}
$$


Applying Lemma 3.4:

$$
\begin{aligned}
= & 1+\sum_{T \in \overline{\mathbb{P}}^{W}} t^{|T|} \theta_{T} \mathcal{V}_{T} \\
& +\sum_{k \geq 2} \frac{1}{k !} \sum_{T \in \overline{\mathbb{T}}^{W}} t^{|T|}\left(\sum_{\substack{\vec{e}=\left(e_{1}, \ldots, e_{k-1}\right) \in E(T)^{k-1} \\
e_{1} \succ \cdots \succ e_{k-1}}} \theta_{T_{\vec{e}, 1}} \cdots \theta_{T_{\vec{e}, k}}\right) \mathcal{V}_{T} \\
= & 1+\sum_{T \in \overline{\mathbb{T}}^{W}} t^{|T|}\left(\theta_{T}+\sum_{k \geq 2} \frac{1}{k !} \sum_{\substack{\vec{e}=\left(e_{1}, \ldots, e_{k-1}\right) \in E(T)^{k-1} \\
e_{1} \succ \cdots \succ e_{k-1}}} \theta_{T_{\vec{e}, 1}} \cdots \theta_{T_{\vec{e}, k}}\right) \mathcal{V}_{T} .
\end{aligned}
$$

Applying Eq. (4.11):

$$
\begin{aligned}
& =1+\sum_{T \in \overline{\mathbb{T}}^{W}} t^{|T|} \mathcal{V}_{T} \\
& =\tilde{g}(t) .
\end{aligned}
$$

Therefore, we get $e^{\tilde{d}(t)}=\tilde{g}(t)$, which is Eq. (2.3) for the system $\Omega_{\mathbb{T}}^{W}$.

To prove Eq. (2.4), first, by Lemma 3.4, we have

$$
\begin{aligned}
\tilde{m}(t) \tilde{g}(t) & =\left(\sum_{T^{\prime} \in \overline{\mathbb{P}}^{W}} \gamma\left(T^{\prime}\right) t^{\left|T^{\prime}\right|-1} \mathcal{V}_{T^{\prime}}\right)\left(\sum_{T^{\prime \prime} \in \overline{\mathbb{T}}^{W}} t^{\left|T^{\prime \prime}\right|} \mathcal{V}_{T^{\prime \prime}}\right) \\
& =\sum_{T \in \overline{\mathbb{T}}^{W}} t^{|T|-1}\left(\sum_{\substack{C \in \mathcal{C}(T) \\
B_{+}\left(P_{C}(T)\right) \in \overline{\mathbb{P}}^{W}}} \gamma\left(B_{+}\left(P_{C}(T)\right)\right) \mathcal{V}_{T}\right. \\
& =\sum_{T \in \overline{\mathbb{T}}^{W}} t^{|T|-1}\left(\sum_{e \in E(T)} \gamma\left(B_{+}\left(P_{e}(T)\right)\right)\right) \mathcal{V}_{T},
\end{aligned}
$$

where the last equality follows from the fact that, for any $C \in \mathcal{C}(T), o\left(B_{+}\left(P_{C}(T)\right)\right)=$ 1 iff $C$ consists of a single edge.

Note that, for any $e \in E(T), \gamma\left(B_{+}\left(P_{e}(T)\right)\right)=w t\left(v_{e}^{\prime}\right)$, where $v_{e}^{\prime}$ is the vertex of $e$ which is further away from the root of $T$. Therefore, continuing with the equation above, we have

$$
\tilde{m}(t) \tilde{g}(t)=\sum_{T \in \overline{\mathbb{T}}^{W}}|T| t^{|T|-1} \mathcal{V}_{T}=\frac{d \tilde{g}(t)}{d t}
$$


Hence, we get Eq. (2.4) for the system $\Omega_{\mathbb{T}}^{W}$.

Finally, we show Eq. (2.5). First, by Lemma 3.4, we have

$$
\begin{aligned}
\tilde{g}(t) \tilde{h}(t) & =\left(\sum_{T^{\prime} \in \overline{\mathbb{T}}^{W}} t^{\left|T^{\prime}\right|} \mathcal{V}_{T^{\prime}}\right)\left(\sum_{T^{\prime \prime} \in \overline{\mathbb{H}}^{W}} \beta\left(T^{\prime \prime}\right) t^{\left|T^{\prime \prime}\right|-1} \mathcal{V}_{T^{\prime \prime}}\right) \\
& =\sum_{T \in \overline{\mathbb{T}}^{W}} t^{|T|-1}\left(\sum_{\substack{C \in \mathcal{C}(T) \\
R_{C}(T) \in \overline{\mathbb{H}}^{W}}} \beta\left(R_{C}(T)\right)\right) \mathcal{V}_{T} .
\end{aligned}
$$

Note that, the set of all admissible cuts $C$ such that $R_{C}(T) \in \overline{\mathbb{H}}^{W}$ is in $1-1$ correspondence with the set of rooted subtrees $S$ of $T$ with $S \in \overline{\mathbb{H}}^{W}$. But any subtree $S$ of $T$ with $S \in \overline{\mathbb{H}}^{W}$ is completely determined by the unique leaf of $S$. Therefore, the set of all admissible cuts $C$ such that $R_{C}(T) \in \overline{\mathbb{H}}^{W}$ is in 1-1 correspondence with the set of non-root vertices of $T$. With this observation, we have, for any $T \in \overline{\mathbb{T}}^{W}$,

$$
\sum_{\substack{C \in \mathcal{C}(T) \\ R_{C}(T) \in \overline{\mathbb{H}}^{W}}} \beta\left(R_{C}(T)\right)=|T| .
$$

Hence, combining the two equations above, we get

$$
\tilde{g}(t) \tilde{h}(t)=\sum_{T \in \overline{\mathbb{T}} W} t^{|T|-1}|T| \mathcal{V}_{T}=\frac{d \tilde{g}(t)}{d t},
$$

which is Eq. (2.5) for the system $\Omega_{\mathbb{T}}^{W}$.

Now, by the universal property of the $\mathcal{N C S}$ system $(\mathcal{N} S y m, \Pi)$ from NCSFs in Theorem 2.6, we have the following correspondence between NCSFs and $W$-labeled rooted trees.

Theorem 4.6 For any nonempty $W \subseteq \mathbb{N}^{+}$, there exists a unique homomorphism $\mathcal{T}_{W}$ : $\mathcal{N} S y m \rightarrow \mathcal{H}_{G L}^{W}$ of graded K-Hopf algebras such that $\mathcal{T}_{W}^{\times 5}(\Pi)=\Omega_{\mathbb{T}}^{W}$.

In particular, we have the following correspondence from the NCSFs in $\Pi$ to the elements in $\Omega_{\mathbb{T}}^{W}$ :

$$
\begin{aligned}
& \mathcal{T}_{W}\left(\Lambda_{m}\right)=\sum_{T \in \overline{\mathbb{S}}_{m}^{W}}(-1)^{o(T)+|T|} \mathcal{V}_{T}, \\
& \mathcal{T}_{W}\left(S_{m}\right)=\sum_{T \in \overline{\mathbb{T}}_{m}^{W}} \mathcal{V}_{T} \\
& \mathcal{T}_{W}\left(\Psi_{m}\right)=\sum_{T \in \overline{\mathbb{H}}_{m}^{W}} \beta_{T} \mathcal{V}_{T}
\end{aligned}
$$




$$
\begin{aligned}
& \mathcal{T}_{W}\left(\Phi_{m}\right)=m \sum_{T \in \overline{\mathbb{P}}_{m}} \theta_{T} \mathcal{V}_{T}, \\
& \mathcal{T}_{W}\left(\Xi_{m}\right)=\sum_{T \in \overline{\mathbb{P}}_{m}} \gamma_{T} \mathcal{V}_{T},
\end{aligned}
$$

for any $m \geq 1$

Proof Note that the coefficients of $t^{m}(m \geq 1)$ of the generating function $\tilde{h}(t)$ (see Eq. (4.3)) are all primitive elements of the Hopf algebra $\mathcal{H}_{G L}^{W}$, since they are linear combinations of chains. Then, by Theorem 2.6, $(b)$, we have a unique homomorphism $\mathcal{T}_{W}: \mathcal{N}$ Sym $\rightarrow \mathcal{H}_{G L}^{W}$ of $K$-Hopf algebras such that $\mathcal{T}_{W}^{\times 5}(\Pi)=\Omega_{\mathbb{T}}^{W}$. In particular, we have $\mathcal{T}_{W}(\lambda(t))=\tilde{f}(t)$ which is same as Eq. (4.21) for any $m \geq 1$. Note that, both sides of Eq. (4.21) have weight $m$ in $\mathcal{N} S y m$ and $\mathcal{H}_{G L}^{W}$, respectively. Also note that the gradings of $\mathcal{N} S y m$ and $\mathcal{H}_{G L}^{W}$ are given by the weights of NCSFs and $W$-labeled rooted trees, respectively, and $\mathcal{N} S y m$ is the free algebra generated by $\Lambda_{m}(m \geq 1)$. By the facts above, it is easy to check that $\mathcal{T}_{W}$ also preserves the gradings.

Note that the graded duals of $\mathcal{N} S y m$ and $\mathcal{H}_{G L}^{W}$ are the graded $K$-Hopf algebras $\mathcal{Q}$ Sym of quasi-symmetric functions and the Connes-Kreimer Hopf algebra $\mathcal{H}_{C K}^{W}$, respectively. Since the $K$-Hopf algebra homomorphism $\mathcal{T}_{W}: \mathcal{N} S y m \rightarrow \mathcal{H}_{G L}^{W}$ preserves the gradings, we can take the graded duals and get the following correspondence.

Corollary 4.7 For any non-empty $W \subseteq \mathbb{N}^{+}, \mathcal{T}_{W}^{*}: \mathcal{H}_{C K}^{W} \rightarrow \mathcal{Q}$ Sym is a homomorphism of graded $K$-Hopf algebras.

Finally, let us end this section with the following two remarks.

Remark 4.8 As we mentioned earlier in Remark 2.3, by applying the specialization $\mathcal{T}_{W}: \mathcal{N} S y m \rightarrow \mathcal{H}_{G L}^{W}$ in Theorem 4.6, we will get a host of identities for the $W$-rooted trees on the right hand sides of Eqs. (4.21)-(4.25) from the identities of the NCSFs on the left hands. We believe some of these identities are also interesting from the aspect of combinatorics of rooted trees. For example, it is not obvious at all that the invariants of rooted trees given by coefficients of the generating functions $\tilde{f}(t), \tilde{d}(t)$, $\tilde{h}(t)$ and $\tilde{m}(t)$ can be obtained by evaluating the corresponding (noncommutative) polynomials of NCSFs at coefficients of $\tilde{g}(t)$ which is just the trivial invariant of rooted trees whose value at any rooted tree is always 1 .

Note that the same problem has been studied in detail in [30] for the differential operators in the $\mathcal{N C S}$ over the differential operator algebras constructed in [29]. But, in order to keep this paper in a certain size, we will skip the discussions on these identities and refer the interested reader to [30] for similar discussions.

Remark 4.9 In the followed paper [31], by using some relations of the $\mathcal{N C S}$ system $\left(\mathcal{H}_{G L}^{W}, \Omega_{\mathbb{T}}^{W}\right)$ with the $\mathcal{N} C S$ systems constructed in [29] over differential operator algebras, it will be shown that, when $W=\mathbb{N}^{+}$, the $K$-Hopf algebra homomorphism $\mathcal{T}_{W}: \mathcal{N} S y m \rightarrow \mathcal{H}_{G L}^{W}$ in Theorem 4.6 is actually an embedding. So, in this case, 
$\mathcal{T}_{W}^{*}: \mathcal{H}_{C K}^{W} \rightarrow \mathcal{Q S y m}$ in Corollary 4.7 is a surjective graded $K$-Hopf algebra homomorphism.

It seems that, the $K$-Hopf algebra homomorphism $\mathcal{T}_{W}: \mathcal{N} S y m \rightarrow \mathcal{H}_{G L}^{W}$ is injective for any non-empty label set $W$. But we will leave this for future investigations.

\section{A combinatorial interpretation of the constants $\theta_{T}$}

In this section, we give a combinatorial interpretation for the constants $\theta_{T}(T \in \mathbb{T})$ in Definition 4.2 which have been used in the construction of $\tilde{d}(t)$ for the $\mathcal{N}$ CS system $\Omega_{\mathbb{T}}^{W}$ over $\mathcal{H}_{G L}^{W}$. We first recall the strict order polynomials and the order polynomials of finite posets (partially ordered sets) (see [23]). Then, by applying some of results proved in [26] for the strict order polynomials of rooted forests and the well-known Reciprocity Relation (see Proposition 5.1) between the strict order polynomials and the order polynomials of finite posets, we show in Proposition 5.8 that, for any $T \in \overline{\mathbb{T}}$, $\theta_{T}$ is same as the coefficient of $s$ of the order polynomial of the rooted forest $B_{-}(T)$.

First, recall that, a poset is a set $P$ with a partial order defined for its elements. Note that, for any unlabeled rooted forest $F \in \mathbb{F}$, the set of the vertices of $F$ has a natural partial order, namely, $u \leq v$ if $u=v$ or $u$ and $v$ are connected by a path with $v$ being further away for the root of the connected component. With this partial order, the set of the vertices of $F$ forms a finite poset, which we will still denote by $F$.

For any $n \geq 1$, let $[n]$ denote the totally ordered set $\{1,2, \ldots, n\}$. For any finite poset $P$, a map $f: P \rightarrow[n]$ is said to be strictly order-preserving (resp. order-preserving) if, for any $a, b \in P$ with $a<b$ in $P$, then $f(a)<f(b)$ (resp. $f(a) \leq f(b))$. It is well-known that, for each finite poset $P$, there exists a unique polynomial $\bar{\Omega}(P, s)$ (resp. $\Omega(P, s)$ ) in formal variable $s$ such that, for any $n \geq 1$, $\bar{\Omega}(P, n)$ (resp. $\Omega(P, n)$ ) equals to the number of strict order-preserving (resp. orderpreserving) from $P$ to $[n]$. The strict order polynomial and the order polynomial of finite posets are related by the so-called reciprocity relation.

Proposition 5.1 (Reciprocity Relation) For any fixed finite poset, we have

$$
\Omega(P, s)=(-1)^{|P|} \bar{\Omega}(P,-s),
$$

where $|P|$ denotes the cardinal number of the finite set $P$.

For a proof of this remarkable result, see Corollary 4.5.15 in [23].

By Corollary 3.15 in [26] (also see Example 4.4 there) and Eq. (5.1) above, we get the (strict) order polynomials of chains and shrubs as follows.

Example 5.2 For any $m \geq 1$, let $C_{m}$ be the chain of hight $m-1$ and $S_{m}$ the shrub with $m$ leaves. Then we have

$$
\begin{aligned}
& \bar{\Omega}\left(C_{m}, s\right)=\left(\begin{array}{c}
s \\
m
\end{array}\right)=\frac{s(s-1) \cdots(s-m+1)}{m !}, \\
& \Omega\left(C_{m}, s\right)=(-1)^{m}\left(\begin{array}{c}
-s \\
m
\end{array}\right)=\left(\begin{array}{c}
s+m-1 \\
m
\end{array}\right),
\end{aligned}
$$




$$
\begin{aligned}
& \bar{\Omega}\left(S_{m}, s\right)=\int_{0}^{s} B_{m}(u) d u=\frac{B_{m+1}(s)-B_{m+1}(0)}{m+1}, \\
& \Omega\left(S_{m}, s\right)=(-1)^{m+1} \int_{0}^{-s} B_{m}(u) d u,
\end{aligned}
$$

where $B_{m}(u)(m \geq 1)$ are the Bernoulli polynomials which are defined by the generating function

$$
\frac{x e^{u x}}{e^{x}-1}=1+\sum_{m=1}^{\infty} B_{m}(u) \frac{x^{m}}{m !} .
$$

\section{Proposition 5.3}

(a) For any finite poset $P, \bar{\Omega}(P, 0)=0$.

(b) For any rooted forest $F=T_{1} T_{2} \cdots T_{m}$ with $T_{i} \in \mathbb{T}(1 \leq i \leq m)$, we have

$$
\bar{\Omega}(F, s)=\bar{\Omega}\left(T_{1}, s\right) \bar{\Omega}\left(T_{2}, s\right) \cdots \bar{\Omega}\left(T_{m}, s\right) .
$$

(c) For any unlabeled rooted tree $T$ with $T=B_{+}(F)$, we have

$$
\begin{aligned}
& \Delta \bar{\Omega}(T, s)=\bar{\Omega}(F, s), \\
& \nabla \Omega(T, s)=\Omega(F, s),
\end{aligned}
$$

where $\Delta$ and $\nabla$ are the linear operators from $K[s] \rightarrow K[s]$ defined by, for any $f(s) \in K[s], \Delta f(s)=f(s+1)-f(s)$ and $\nabla f(s)=f(s)-f(s-1)$, respectively.

(a) is well-known, for example, it can be easily proved by the recurrent formulas in [22] for the (strict) order polynomials. (b) follows directly from the definition of the strict order polynomials. (c) was first proved by J. Shareshian (unpublished). For a proof of Eq. (5.7), see Theorem 4.5 in [26]. Eq. (5.8) can be proved similarly as Eq. (5.7). For more studies on these properties of the (strict) order polynomials, see [27] and [22].

Now, for any finite poset $P$, we define

$$
\begin{aligned}
\phi_{P} & :=\left.\frac{d}{d s} \bar{\Omega}(P, s)\right|_{s=0}, \\
\varphi_{P} & :=\left.\frac{d}{d s} \Omega(P, s)\right|_{s=0} .
\end{aligned}
$$

By Proposition 5.1 and 5.3, it is easy to see that we have the following corollary.

\section{Corollary 5.4}

(a) For any finite poset $P$, we have $\phi_{P}=(-1)^{|P|-1} \varphi_{P}$.

(b) For any rooted forest $F \in \mathbb{F}$ with more than one connected component, we have $\phi_{F}=\varphi_{F}=0$. 
The following proposition have been proved in [26]. But note that the definition of $T_{\vec{e}, k}$ we adapt here is different form the one used in [26]. So the equations in the proposition below have been modified accordingly.

\section{Proposition 5.5}

(a) The constants $\left\{\phi_{T} \mid T \in \mathbb{T}\right\}$ satisfy, and are uniquely determined by

$$
\begin{aligned}
& \phi_{T=0}=1, \\
& \phi_{T}=-\sum_{k=2}^{v(T)} \frac{1}{k !} \sum_{\substack{\vec{e}=\left(e_{1}, \ldots, e_{k-1}\right) \in E(T)^{k-1} \\
e_{1} \succ \cdots \succ e_{k-1}}} \phi_{B_{-}\left(T_{\vec{e}, 1}\right) \cdots \phi_{B_{-}\left(T_{\vec{e}, k-1}\right)} \phi_{T_{\vec{e}, k}},},
\end{aligned}
$$

when $v(T) \geq 2$.

(b) For any $T \in \mathbb{T}$, we have

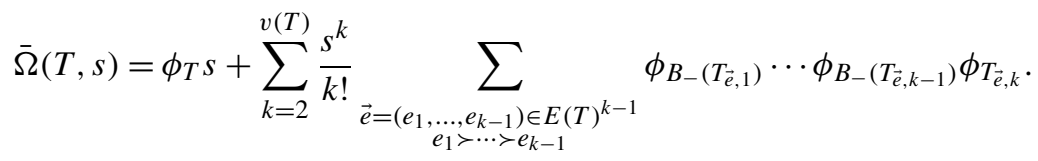

By Proposition 5.5, Corollary 5.4, (a) and Proposition 5.1, it is easy to check that the order polynomials $\Omega(T, s)$ of rooted trees $T$ can be obtained as follows.

\section{Corollary 5.6}

(a) The constants $\left\{\varphi_{T} \mid T \in \mathbb{T}\right\}$ satisfy and are uniquely determined by

$$
\begin{aligned}
& \varphi_{T=\circ}=1, \\
& \varphi_{T}=\sum_{k=2}^{v(T)} \frac{(-1)^{k}}{k !} \sum_{\substack{\vec{e}=\left(e_{1}, \ldots, e_{k-1}\right) \in E(T)^{k-1} \\
e_{1} \succ \cdots \succ e_{k-1}}} \varphi_{B_{-}\left(T_{\vec{e}, 1}\right)} \cdots \varphi_{B_{-}\left(T_{\vec{e}, k-1}\right)} \varphi_{T_{\vec{e}, k}}
\end{aligned}
$$

when $v(T) \geq 2$.

(b) For any $T \in \mathbb{T}$, we have

$$
\Omega(T, s)=\varphi_{T} s+\sum_{k=2}^{v(T)} \frac{s^{k}}{k !} \sum_{\substack{\vec{e}=\left(e_{1}, \ldots, e_{k-1}\right) \in E(T)^{k-1} \\ e_{1} \succ \cdots \succ e_{k-1}}} \varphi_{B_{-}\left(T_{\vec{e}, 1}\right)} \cdots \varphi_{B_{-}\left(T_{\vec{e}, k-1}\right)} \varphi_{T_{\vec{e}, k}} .
$$

Note that, from the definition of the order polynomials, we have $\Omega(P, 1)=1$ for any finite poset $P$. Using this fact and evaluating $\Omega(T, s)$ in Eq. (5.11) at $s=1$, we get another recurrent formula for the constants $\varphi_{T}$ of rooted trees. 
Corollary 5.7 The constants $\left\{\varphi_{T} \mid T \in \mathbb{T}\right\}$ satisfy and are uniquely determined by

$$
\begin{aligned}
& \varphi_{T=\circ}=1, \\
& \varphi_{T}=1-\sum_{k=2}^{v(T)} \frac{1}{k !} \sum_{\substack{\vec{e}=\left(e_{1}, \ldots, e_{k-1}\right) \in E(T)^{k-1} \\
e_{1} \succ \cdots \succ e_{k-1}}} \varphi_{B_{-}\left(T_{\vec{e}, 1}\right)} \cdots \varphi_{B_{-}\left(T_{\vec{e}, k-1}\right)} \varphi_{T_{\vec{e}, k}} .
\end{aligned}
$$

Now, we consider the constants $\theta_{T}(T \in \mathbb{T})$ defined in Definition 4.2 in Sect. 4 and prove the following main result of this section.

Proposition 5.8 For any $T \in \mathbb{T}$ with $T=B_{+}(F)$, we have

$$
\theta_{T}=\varphi_{F}
$$

Proof For any $T \in \mathbb{T}$ with $T=B_{+}(F)$, we set $\tilde{\theta}_{T}:=\varphi_{F}$. For convenience, we also set $\tilde{\theta}_{\circ}=0$. We need show that $\tilde{\theta}_{T}=\theta_{T}$ for any $T \in \mathbb{T}$.

Note first that, if $T$ the singleton $\circ$ or a non-primitive rooted tree, i.e. $F$ is the empty or has at least two connected components, by the definition of $\theta_{T}$ in Definition 4.2 and Corollary 5.4, $(b)$, we have $\theta_{T}=\tilde{\theta}_{T}=0$. To show that $\tilde{\theta}_{T}=\theta_{T}$ for all primitive rooted trees $T$, it will be enough to show that, $\tilde{\theta}_{T}(T \in \mathbb{P})$ also satisfies the recurrent relations in Definition 4.2.

We use the mathematical induction on $v(T)$. First, for the case $v(T)$, i.e. $T=$ $B_{+}(\circ)=C_{2}$. Since $\Omega(\circ, s)=s, \tilde{\theta}_{T}=\varphi_{\circ}=1$. While $\theta_{T}$ is defined to be 1 in Definition 4.2. Hence, $\theta_{T}=\tilde{\theta}_{T}$ in this case.

Now, assume $T=B_{+}\left(T^{\prime}\right)$ with $T^{\prime} \in \mathbb{T}$ and $v\left(T^{\prime}\right) \geq 2$. Applying Eq. (5.12) to $T^{\prime}$, we have

$$
\varphi_{T^{\prime}}=1-\sum_{k=2}^{v\left(T^{\prime}\right)} \frac{1}{k !} \sum_{\substack{\vec{e}=\left(e_{1}, \ldots, e_{k-1}\right) \in E\left(T^{\prime}\right)^{k-1} \\ e_{1} \succ \cdots \succ e_{k-1}}} \varphi_{B_{-}\left(T_{\vec{e}, 1}\right)} \cdots \varphi_{B_{-}\left(T_{\vec{e}, k-1}\right)} \varphi_{T_{\vec{e}, k}} .
$$

Note that, we can identify the set of $\vec{e}=\left(e_{1}, \ldots, e_{k-1}\right) \in E\left(T^{\prime}\right)^{k-1}$ with the set of $\vec{e}=\left(e_{1}, \ldots, e_{k-1}\right) \in E(T)^{k-1}$ such that $T_{\vec{e}, k} \neq \circ$. With these observations, by replacing the constants $\varphi$ 's by $\tilde{\theta}$ 's in the summation of the equation above, we have

$$
\tilde{\theta}_{T}=1-\sum_{k=2}^{v(T)} \frac{1}{k !} \sum_{\substack{\vec{e}=\left(e_{1}, \ldots, e_{k-1}\right) \in E(T)^{k-1} \\ e_{1} \succ \cdots \succ e_{k-1} \\ T_{\vec{e}, k} \neq 0}} \tilde{\theta}_{T_{\vec{e}, 1}} \tilde{\theta}_{T_{\vec{e}, 2}} \cdots \tilde{\theta}_{T_{\vec{e}, k}} .
$$


Applying the induction assumption to $T_{\vec{e}, j}$ 's and using the fact that $\tilde{\theta}_{\circ}=0$ :

$$
=1-\sum_{k=2}^{v(T)} \frac{1}{k !} \sum_{\substack{\vec{e}=\left(e_{1}, \ldots, e_{k-1) \in E(T)^{k-1}} \\ e_{1} \succ \cdots \succ e_{k-1}\right.}} \tilde{\theta}_{T_{\vec{e}, 1}} \tilde{\theta}_{T_{\vec{e}, 2}} \cdots \tilde{\theta}_{T_{\vec{e}, k}} .
$$

Therefore, the constants $\left\{\tilde{\theta}_{T} \mid T \in \mathbb{P}\right\}$ also satisfy the recurrent relations of $\left\{\theta_{T} \mid\right.$ $T \in \mathbb{P}\}$ in Definition 4.2. Hence, we have $\theta_{T}=\tilde{\theta}_{T}=\varphi_{T}$ for any $T \in \mathbb{T}$.

For an interpretation of the constant $\phi_{T}$, which we have shown is same as $(-1)^{v(T)-1} \varphi_{T}=(-1)^{v(T)-1} \theta_{B_{+}(T)}$, in terms of the numbers of chains with fixed lengths in the lattice of the ideals of the poset $T$, see Lemma 2.8 in [22].

Corollary 5.9 For any $T \in \mathbb{P}$, we have

$$
\nabla \Omega(T, s)=\theta_{T} s+\sum_{k=2}^{v(T)} \frac{s^{k}}{k !} \sum_{\substack{\vec{e}=\left(e_{1}, \ldots, e_{k-1}\right) \in E(T)^{k-1} \\ e_{1} \succ \cdots \succ e_{k-1}}} \theta_{B_{-}\left(T_{\vec{e}, 1}\right)} \cdots \theta_{B_{-}\left(T_{\vec{e}, k-1}\right)} \theta_{T_{\vec{e}, k}} .
$$

In particular, $\theta_{T}$ is also the coefficient of $s$ of the polynomial $\nabla \Omega(T, s)$.

Proof First, we write $T=B_{+}\left(T^{\prime}\right)$ with $T^{\prime} \in \mathbb{T}$. By Eq. (5.8) and Proposition 5.5, (b), we have

$$
\begin{aligned}
\nabla \Omega(T, s) & =\Omega\left(T^{\prime}, s\right) \\
& =\varphi_{T^{\prime}} s+\sum_{k=2}^{v\left(T^{\prime}\right)} \frac{s^{k}}{k !} \sum_{\substack{\vec{e}=\left(e_{1}, \ldots, e_{k-1}\right) \in E\left(T^{\prime}\right)^{k-1} \\
e_{1} \succ \cdots \succ e_{k-1}}} \varphi_{B_{-}\left(T_{\vec{e}, 1}\right)} \cdots \varphi_{B_{-}\left(T_{\vec{e}, k-1}\right)} \varphi_{T_{\vec{e}, k}^{\prime}} .
\end{aligned}
$$

Then, applying Eq. (5.13) and replacing the constants $\varphi$ 's in the sum above by the constant $\theta$ 's, we get Eq. (5.14).

Acknowledgements The author is very grateful to both referees for many invaluable suggestions to improve the paper. In particular, all the diagrams of trees in this paper are due to one of the referees who very kindly sent the author all the latex commands.

\section{References}

1. Abe, E.: Hopf Algebras. Translated from the Japanese by Hisae Kinoshita and Hiroko Tanaka. Cambridge Tracts in Mathematics, vol. 74. Cambridge University Press, Cambridge (1980)

2. Aguiar, M., Bergeron, N., Sottile, F.: Combinatorial Hopf algebras and generalized DehnSommerville relations. Compos. Math. 142(1), 1-30 (2006). See also math.CO/0310016

3. Bass, H., Connell, E., Wright, D.: The Jacobian conjecture, reduction of degree and formal expansion of the inverse. Bull. Am. Math. Soc. 7, 287-330 (1982) 
4. Connes, A., Kreimer, D.: Hopf algebras, renormalization and noncommutative geometry. Commun. Math. Phys. 199(1), 203-242 (1998). See also hep-th/9808042

5. Duchamp, G., Klyachko, A., Hivert, H., Thibon, J.-Y.: Noncommutative symmetric functions. VI. Free quasi-symmetric functions and related algebras. Int. J. Algebra Comput. 12(5), 671-717 (2002)

6. Duchamp, G., Klyachko, A., Krob, D., Thibon, J.-Y.: Noncommutative symmetric functions, III: deformations of Cauchy and convolution algebras, Lie computations. Discrete Math. Theor. Comput. Sci. 1(1), 159-216 (1997)

7. van den Essen, A.: Polynomial Automorphisms and the Jacobian conjecture. Progress in Mathematics, vol. 190. Birkhäuser, Basel (2000)

8. Foissy, L.: Les algèbres de Hopf des arbres enracinés décorés, I. Bull. Sci. Math. 126(3), 193-239 (2002)

9. Foissy, L.: Les algèbres de Hopf des arbres enracinés décorés, II. Bull. Sci. Math. 126(4), 249-288 (2002). See also math.QA/0105212

10. Gelfand, I.M., Krob, D., Lascoux, A., Leclerc, B., Retakh, V.S., Thibon, J.-Y.: Noncommutative symmetric functions. Adv. Math. 112(2), 218-348 (1995). See also hep-th/9407124

11. Gessel, I.: Multipartite $P$-partitions and inner products of skew Schur functions. Comtemp. Math. 34, 289-301 (1984)

12. Grossman, R., Larson, R.G.: Hopf-Algebraic structure of families of Trees. J. Algebra 126(1), 184210 (1989)

13. Hoffman, M.E.: Combinatorics of rooted trees and Hopf algebras. Trans. Am. Math. Soc. 355(9), 3795-3811 (2003). See also math.CO/0201253

14. Knutson, D.: $\lambda$-Rings and the Representation Theory of the Symmetric Groups. Lecture Notes in Mathematics, vol. 308. Springer, Berlin (1973)

15. Kreimer, D.: On the Hopf algebra structure of perturbative quantum field theories. Adv. Theor. Math. Phys. 2.2, 303-334 (1998). See also math.QA/9707029

16. Krob, D., Leclerc, B., Thibon, J.-Y.: Noncommutative symmetric functions, II: transformations of alphabets. Int. J. Algebra Comput. 7(2), 181-264 (1997)

17. Krob, D., Thibon, J.-Y.: Noncommutative symmetric functions, IV: quantum linear groups and Hecke algebras at $q=0$ J. Algebr. Comb. 6(4), 339-376 (1997)

18. Krob, D., Thibon, J.-Y.: Noncommutative symmetric functions, V: a degenerate version of $U_{q}\left(\mathrm{gl}_{N}\right)$. Int. J. Algebra Comput. 9(3-4), 405-430 (1999)

19. Macdonald, I.G.: Symmetric Functions and Hall Polynomials. With contributions by A. Zelevinsky, 2nd edn. Oxford Mathematical Monographs. Oxford Science Publications. Clarendon/Oxford University Press, New York (1995)

20. Malvenuto, C., Reutenauer, C.: Duality between quasi-symmetric functions and the Solomon descent algebra. J. Algebra 177(3), 967-982 (1995)

21. Montgomery, S.: Hopf Algebras and Their Actions on Rings. CBMS Regional Conference Series in Mathematics, vol. 82. American Mathematical Society, Providence (1993)

22. Shareshian, J., Wright, D., Zhao, W.: A new approach to order polynomials of labeled posets and their generalizations, math.CO/0311426

23. Stanley, R.P.: Enumerative Combinatorics I. Cambridge University Press, Cambridge (1997)

24. Stanley, R.P.: Enumerative Combinatorics II. Cambridge University Press, Cambridge (1999)

25. Thibon, J.-Y.: Lectures on noncommutative symmetric functions. In: Interaction of Combinatorics and Representation Theory. MSJ Memories, vol. 11, pp. 39-94. Math. Soc. Japan, Tokyo (2001)

26. Wright, D., Zhao, W.: D-log and formal flow for analytic isomorphisms of $n$-space. Trans. Am. Math. Soc. 355(8), 3117-3141 (2003). See also math.CV/0209274

27. Zhao, W.: A family of invariants of rooted forests. J. Pure Appl. Algebra 186(3), 311-327 (2004). See also math.CO/0211095

28. Zhao, W.: Noncommutative symmetric systems over associative algebras. J. Pure Appl. Algebra 210(2), 363-382. See also math.CO/0509133

29. Zhao, W.: Differential operator specializations of noncommutative symmetric functions. Adv. Math. 214(2), 639-665. See also math.CO/0509134

30. Zhao, W.: Noncommutative symmetric functions and the inversion problem. math.CV/0509135. Submitted

31. Zhao, W.: $\mathcal{N C S}$ systems over differential operator algebras and the Grossman-Larson Hopf algebra of labeled rooted trees. Preprint. math.CO/0509138. Submitted 